\title{
Etiology, Diagnostic Testing, and Dermatology Consultation Utilization in Skin Infections in Hematopoietic Stem Cell Transplant Patients: A 5-year Review
}

\author{
Lymaries Velez ${ }^{1}$, Steven Toffel ${ }^{1}$, Elizabeth Tremblay ${ }^{2}$, Kairav Shah $^{3}$, Jena Auerbach ${ }^{4}$, Kenneth Rand ${ }^{3,4}$, \\ Kathryn Anne Potter ${ }^{5}$, Stacy G. Beal ${ }^{4}$ \\ ${ }^{1}$ University of Florida College of Medicine; Gainesville, FL, USA \\ ${ }^{2}$ UF Health Shands Hospital, Department of Infection Control, Gainesville, FL, USA \\ ${ }^{3}$ University of Florida, Department of Internal Medicine, Division of Infectious Diseases and Global \\ Medicine, Gainesville, FL, USA \\ ${ }^{4}$ University of Florida, Department of Pathology, Gainesville, FL, USA \\ ${ }^{5}$ University of Florida, Department of Dermatology, Gainesville, FL, USA
}

\begin{abstract}
Objectives: Hematopoietic stem cell transplantation (HSCT) patients are at higher risk of infection compared to their healthy counterparts. Aims were identity type and incidence of skin infections, describe the use of diagnostic tests, and identify the role of dermatology consults.
\end{abstract}

Methods: In this single institution retrospective chart review, data were extracted from the medical record. A clinically diagnosed skin infection was defined as any skin pathology treated with antibiotics, antivirals, antifungals, or antiparasitics. The diagnostic tests data such as cultures, polymerase chain reactions (PCR), and direct fluorescent antibody (DFA) tests, and dermatology consultations were also collected.

Results: A total of 92 patients and 143 skin infections were identified in the 5-year study period. The majority of infections occurred while the patient was not neutropenic. The infectious agents responsible for infections varied depending on a patient's age, neutropenia status, graft-versus-host disease (GVHD) diagnosis, and transplant type. Only 25 infections (17\%) received a dermatology consultation. On average, infections associated with dermatology consultation received a higher number of diagnostic tests compared to those that did not receive a dermatology consultation.

Conclusions: The etiologies and severities of clinically identified skin infections in HSCT patients are varied and require continuous vigilance of dermatological health. Dermatologists are not necessarily the physician's ordering more diagnostic. Therefore, earlier assessment by dermatologists might prevent excessive laboratory testing and earlier management of severe clinically identified infections. J Microbiol Infect Dis 2019; 9(2):59-67.

Keywords: Skin infections; Hematopoietic Stem Cell Transplantation; Dermatology Consultation; Neutropenia

\section{INTRODUCTION}

Hematologic disease patients receiving stem cell transplants are more susceptible to infections relative to their healthy counterparts [1-3]. Hematopoietic stem cell transplantation (HSCT) is indicated for cancers such as leukemia, lymphoma, and multiple myeloma [4]. Patients with other diseases such as aplastic anemia, myelodysplastic syndrome, and even brain tumors [5] can also potentially benefit from HSCT. Many of these diseases affect the formation, proliferation, and function of the immune system [6]. Preparation for an HSCT further lowers the immune response and engraftment following a transplant can be prolonged, further setting the patient up for infection [7, 8]. Immune system impairment can last for some time following the SCT treatment, from a few months after an autologous transplantation to one to two years after an allogenic or syngeneic transplantation [1,4]. Therefore, risk for infection is not limited to the time during or immediately after the transplantation occurs. There are three main types of stem cell transplants: autologous, 
allogeneic, and syngeneic [4,7], each with their own set of risks (including varying risks for graftversus-host disease [GVHD]) and benefits.

The skin serves as the first defense barrier of the immune system, and therefore skin infections encompass a wide variety of etiologies and severity $[9,10]$. Possible skin infections in HSCT patients include fungal, bacterial, viral, and parasitic infections [11-14]. These skin pathologies may have atypical presentations in immunocompromised patients, making a dermatology consultation an important consideration [15]. Dermatologists have been shown to more accurately diagnose skin pathologies compared to generalists, and diagnoses often change after consultation [16]. In one pediatric study, hematology-oncology was the department with the second highest requests for dermatology consultation [17]. Therefore, dermatology consultation leads to improved diagnostic accuracy and avoids unnecessary treatments, which in turn can potentially lead to cost savings [16]. However, excluding large academic or teaching institutions, most hospitals only have dermatologists available as consultants rather than direct inpatient teams [15]. This study aimed to (1) identity the type and incidence of skin infections, (2) describe the use of diagnostic tests for infectious class identification, and (3) identify the role and prevalence of dermatology consults in diagnosis of skin infections.

\section{METHODS}

This single-institution retrospective chart review was performed at the University of Florida Health Hospital after Institutional Review Board approval and included patients with autologous or allogeneic hematopoietic stem cell transplant (HSCT) and at least one clinically diagnosed skin infection between January 2012 to December 2016. In this study, a clinically diagnosed skin infection is defined as any skin pathology that was ultimately treated with antibiotics, antivirals, antifungals, or antiparasitics. These include skin pathologies that were treated empirically, those that had positive diagnostic testing, and those that had negative diagnostic testing but were still treated with antibiotics, antivirals, antifungals, or antiparasitics. Any skin pathology treated with an alternative medication, such as steroids, for example, was not included in this study. These clinically diagnosed skin infections were identified by the following criteria: referral to inpatient or outpatient dermatology, admitting diagnosis of "infection," "skin," "rash," "abscess," "derm," "cutaneous," or "infected"; skin biopsy submitted to pathology or culture; swab submitted for culture; cultures with source "skin"; or skin specimen sent for Varicella-Zoster Virus or Herpes Simplex Virus Polymerase Chain Reaction (PCR) or Direct Fluorescent Antibody (DFA). Other inclusion criteria for this study included formal diagnoses for superficial epidermal infections, including impetigo and folliculitis, and for deeper dermal infections, including cellulitis, erysipelas, furuncles and carbuncles. Demographics and information concerning the transplant and the nature and circumstances of these clinically diagnosed skin infections was collected from the electronic health record.

Diagnostic tests and clinical judgement used to identify and manage the infections were also collected. This included the location where the patient first presented with the skin infection as well as the physical location of the skin lesions. The patient was determined to have a dermatology consultation if he or she received one while in the hospital or if he or she went directly to a dermatology outpatient clinic for infection management. Neutropenia was defined as $<1500$ neutrophils/microliter on the day the patient presented with the initial complaint. Lastly, diagnostic test performed for a particular infection event included bacterial culture, viral culture, fungal culture, acid-fast bacilli (AFB) culture, anaerobic culture, direct stain (e.g. gram, AFB, fungal, etc.), Tzanck test, Herpes Simplex Virus - fluorescent antibody (HSV-FA), Varicella Zoster Virus (VZV-FA), Herpes Simplex Virus Polymerase Chain Reaction (HSV PCR), Varicella Zoster Virus Polymerase Chain Reaction (VZV PCR), biopsy/histopathology, and Other.

Study data were collected and managed using REDCap electronic data capture tools hosted at University of Florida [18]. REDCap (Research Electronic Data Capture) is a secure, web-based application designed to support data capture for research studies, providing: 1) an intuitive interface for validated data entry; 2) audit trails 
for tracking data manipulation and export procedures; 3) automated export procedures for seamless data downloads to common statistical packages; and 4) procedures for importing data from external sources. Further descriptive statistical analysis was conducted using Statistical Analysis Software (SAS) and Excel. Ttests were used to compare continuous variables (e.g. age) whereas Fisher's exact test was used to compare categorical variables.

\section{RESULTS}

A total of 92 patients were included, resulting in 143 individual skin infections. Baseline characteristics are summarized in Table 1. A total of 60 patients $(65.2 \%)$ received an allogeneic HSCT and 32 patients (34.8\%) received autologous HSCT. Allogeneic transplant patients had a mean age of 43.9 years and autologous transplant patients had a mean age of 49.7 years. This difference was not statistically significant $(p=0.210)$. A total of 26allogeneic transplant patients were male (43.3\%) as opposed to 21 autologous transplant patients $(65.6 \%)(p=0.051)$.

Allogeneic transplant patients had a higher proportion of skin infections (90/143) (Table 2). However, autologous transplant patients had a higher number of skin infections per patient on average, 1.66 infections compared with 1.43 infections in allogeneic transplant patients ( $p$ value $=0.1485$ ).

The 143 infections were assessed for age at infection onset ( $\leq 18$ yrs. vs $>18$ yrs.), neutropenia presence, GVHD diagnosis, and transplant type (Table 2). There were no significant differences between pediatrics $(\leq 18$ yrs) and adults ( $>18 \mathrm{yrs}$.) with regards to the proportion of infections caused by various organisms (Table 2). A total of 98 (68.5\%) infections occurred when no neutropenia was detected at infection onset (98/143). Infections were evenly split between GVHD diagnoses: $72 / 143(50.4 \%)$ occurred in those with GVHD, and $71 / 143(49.7 \%)$ occurred in those without. For Tables 2 and 3 categories of "single bacterial pathogen," "identified bacterial organism - cellulitis associated," and "mixed bacterial infection," organisms identified included Klebsiella pneumoniae $(\mathrm{n}=4)$, Corynebacterium $(n=1)$, Methicillin-sensitive Staphylococcus aureus $\quad(\mathrm{n}=12), \quad$ Methicillin-resistant Staphylococcus aureus $(\mathrm{n}=15)$, Enterococcus faecalis $(n=2), \quad$ Vancomycin resistant Enterococcus faecium $(\mathrm{n}=4)$, Proteus mirabilis $(n=1)$, Propionibacterium $(n=1)$, Acinetobacter baumannii $(n=3)$, Enterobacter cloacae $(n=1)$, Stenotrophomonas maltophilia $(n=1)$, Staphylococcus non-aureus $(\mathrm{n}=2)$, Streptococcus pyogenes/Group A Streptecoccus $(\mathrm{n}=2)$, Group B Streptococcus $(\mathrm{n}=3)$, Pseudomonas aeruginosa $(n=4)$, Escherichia coli $(\mathrm{n}=5)$.

The etiology of infections remained unknown in 24 cases with allogeneic transplants $(27 \%)$ and 20 cases with autologous transplants (38\%) (Table 2). Similarly, the etiology of infections in those with GVHD remained unknown in 19 cases $(26 \%)$ and 25 cases in those without GVHD (35\%). Additionally, 25 patients with allogeneic transplants had Herpes (HSV/VZV) infections $(28 \%)$ compared to 5 patients with autologous transplants (9\%) p-value $=0.0104$. The same applies to patients with GVHD (20/72, $28 \%)$ when compared to no GVHD (10/71, 14\%) however this difference was not statistically significant $(p=0.064)$.

In Table 3, infections are separated by infectious class and engraftment period. There were no statistically significant differences between the infectious class and the engraftment period during which the infection presented (all pvalues $>0.05$ ). Engraftment was defined as the period when new health cells begin to develop to replace old malignant blood cells.

A total of $17 \%$ of patients received dermatology consultations $(n=25)$, which included direct dermatologic management and hospital dermatology consultations. A total of $32 \%$ of infections remained unidentified with no dermatology consultation compared to $24 \%$ with dermatology consultation. The study also recorded the location where infections initially presented: $14.7 \%$ at the head/neck, $5.9 \%$ perioral, $14.7 \%$ trunk, $11.8 \%$ upper extremities, $12.4 \%$ genitals, $4.7 \%$ perianal/buttocks, $9.4 \%$ surgical site, $2.4 \%$ central or peripheral venous catheter placement sites, $18.2 \%$ lower extremities, and $5.9 \%$ feet.

The most utilized diagnostic test was a bacterial culture $(n=68)$, followed by viral cultures $(n=20)$ 
and HSV-FA $(\mathrm{n}=18)$. Infections associated with a dermatology consult performed more tests (1.52) compared to those that were not associated with a dermatology consult (1.31).

\section{DISCUSSION}

The majority of the 143 infections occurred while the patient was not neutropenic $(98 / 143,68.5 \%)$. Generally, neutropenia following transplantation is a serious risk factor for infection [1], but the majority of our infections occurred after this period of immunosuppression. A previous study demonstrated that after 2 weeks of neutropenia, a patient's risk of fungal infections increases [19]. However, only 11 of the 143 infections were associated with a fungus. The main risk factor for fungal infections in oncology patients is the presence of severe neutropenia and most of our population was not neutropenic at infection onset $(98 / 143,68.5 \%)$, so the low number of fungal infections is understandable, as seen in Table 2.

HSCT patients and immunocompetent patients present similarly with dermatophyte infections [20]. Interestingly, while Candida and Pseudomonas are often found together as a mixed infection, their interactions are antagonistic [21]. Candida and Group B Streptococcus (GBS) was another mixed infection found in our population. Group B Streptococcus generally causes most severe infections in neonates, but can also be found in patients with underlying medical conditions. Invasive candidiasis is normally associated with neutropenia. However, while our patient was not neutropenic at infection onset, perhaps a GBS infection allowed for the subsequent growth of a Candida infection as well [22]. Fusarium infections have a mortality rate of $80 \%$ in neutropenic patients and is, therefore, an organism that needs to be identified early on [20]. It is one of the most common causes of fungemia, and $75 \%$ of Fusarium fungemia exhibit cutaneous manifestations, making it important for clinicians to monitor a patient's dermatological health [20]. It is vital to note that skin biopsies are necessary to conclude tissue invasion since a positive culture alone does not indicate invasive infection [20]. Because our patient was not neutropenic at infection onset and only received a bacterial and fungal culture, it is possible that the patient only had a localized infection rather than a skin manifestation of a disseminated fungal infection [20].

Table 1. Study population demographics and reason for stem cell transplantation.

\begin{tabular}{|c|c|c|c|c|c|}
\hline \multirow{2}{*}{$\frac{\text { Features of Patients }}{\text { Primary diagnosis, } n}$} & \multirow{2}{*}{$\begin{array}{l}\text { All } \\
\text { All }\end{array}$} & \multicolumn{2}{|c|}{ Allo-HSCT } & \multicolumn{2}{|c|}{ Auto-HSCT } \\
\hline & & $\leq 18$ Peds & $>18$ Adults & $\leq 18$ Peds & $>18$ Adults \\
\hline Acute Lymphoblastic Leukemia & 10 & 1 & 8 & - & 1 \\
\hline Acute Myeloid Leukemia & 22 & 2 & 20 & - & - \\
\hline Aplastic Anemia & 3 & 1 & 2 & - & - \\
\hline Bone sarcoma & 1 & - & 1 & - & - \\
\hline Brain tumor & 2 & - & - & 2 & - \\
\hline Chronic Lymphocytic Leukemia & 2 & - & 2 & - & - \\
\hline Chronic Myeloid Leukemia & 3 & 1 & 2 & - & - \\
\hline Hodgkin Lymphoma & 8 & - & 4 & - & 4 \\
\hline Hurler's Syndrome & 1 & - & - & 1 & - \\
\hline Kostmann's Syndrome & 1 & 1 & - & - & - \\
\hline Mixed-Phenotype Acute Leukemia & 2 & 1 & 1 & - & - \\
\hline Multiple Myeloma & 15 & - & - & - & 15 \\
\hline Myelodysplastic Syndrome & 7 & - & 7 & - & - \\
\hline Myelofibrosis & 2 & - & 2 & - & - \\
\hline Neuroblastoma & 2 & - & - & 2 & - \\
\hline Non-Hodgkin Lymphoma & 10 & - & 3 & - & 7 \\
\hline SCID & 1 & 1 & - & - & - \\
\hline All & 92 & 8 & 52 & 5 & 27 \\
\hline
\end{tabular}

Allo=Allogeneic, Auto=Autologous, $\mathrm{HSCT}=$ Hematopoietic stem cell transplantation, Peds=Pediatrics 
Table 2: Proportion of organism by age, neutropenic status, GVHD diagnosis, and transplant type.

\begin{tabular}{|c|c|c|c|c|c|c|c|c|c|c|c|c|c|c|}
\hline \multirow[b]{2}{*}{ Organism } & \multirow[b]{2}{*}{ Total } & \multicolumn{3}{|c|}{$\begin{array}{l}\text { Infections by Age } \\
\text { n (column \%) }\end{array}$} & \multicolumn{4}{|c|}{$\begin{array}{l}\text { Neutropenia Present }{ }^{3} \\
\text { n (column \%) }\end{array}$} & \multicolumn{3}{|c|}{$\begin{array}{l}\text { GVHD Present } \\
\text { n (column \%) }\end{array}$} & \multicolumn{3}{|c|}{$\begin{array}{l}\text { Transplant Type } \\
\text { n (column \%) }\end{array}$} \\
\hline & & $\leq 18$ & $>18$ & $\begin{array}{c}\text { p- } \\
\text { value* }\end{array}$ & Yes & No & Unk & $\begin{array}{c}\text { p-value* } \\
\text { (excl } \\
\text { unk) }\end{array}$ & Yes & No & $\begin{array}{c}\text { p- } \\
\text { value* }\end{array}$ & Allo & Auto & $\begin{array}{c}\text { p- } \\
\text { value }^{*}\end{array}$ \\
\hline Herpes (HSV/VZV) & 30 & $2(10.5)$ & $28(22.6)$ & 0.3645 & $7(20.6)$ & $20(20.4)$ & $3(27.3)$ & 1.000 & $20(27.8)$ & $10(14.1)$ & 0.0636 & $25(27.8)$ & $5(9.4)$ & 0.0104 \\
\hline Single bacterial pathogen ${ }^{\top}$ & 27 & $5(26.3)$ & $22(17.7)$ & 0.3582 & $7(20.6)$ & 17 (17.3) & $3(27.3)$ & 0.7966 & $14(19.4)$ & $13(18.3)$ & 1.000 & $17(18.9)$ & $10(18.9)$ & 1.000 \\
\hline $\begin{array}{l}\text { Unknown organism - } \\
\text { noncellulitis associated }\end{array}$ & 25 & $5(26.3)$ & $20(16.1)$ & 0.3286 & $5(14.7)$ & $18(18.4)$ & $2(18.2)$ & 0.7948 & $9(12.5)$ & $16(22.5)$ & 0.1283 & $12(13.3)$ & $13(24.5)$ & 0.1114 \\
\hline $\begin{array}{l}\text { Unidentified organism - } \\
\text { cellulitis associated }^{2}\end{array}$ & 19 & $2(10.5)$ & $17(13.7)$ & 1.000 & $7(20.6)$ & $12(12.2)$ & -- & 0.2609 & $10(13.9)$ & $9(12.7)$ & 1.000 & $12(13.3)$ & $7(13.2)$ & 1.000 \\
\hline $\begin{array}{l}\text { Identified bacterial organism - } \\
\text { cellulitis associated }\end{array}$ & 13 & -- & $13(10.5)$ & -- & $3(8.8)$ & $9(9.2)$ & $1(9.1)$ & 1.000 & $8(11.1)$ & $5(7.0)$ & 0.5625 & $7(7.8)$ & $6(11.3)$ & 0.5515 \\
\hline Mixed bacterial infection ${ }^{1}$ & 10 & $2(10.5)$ & $8(6.5)$ & 0.6225 & $1(2.9)$ & $9(9.2)$ & -- & -- & $4(5.6)$ & $6(8.5)$ & 0.5324 & $6(6.7)$ & $4(7.5)$ & 1.000 \\
\hline Dermatophyte & 5 & $1(5.3)$ & $4(3.2)$ & -- & $1(2.9)$ & $4(4.1)$ & -- & -- & $1(1.4)$ & $4(5.6)$ & -- & $2(2.2)$ & $3(5.7)$ & -- \\
\hline HPV & 4 & $1(5.3)$ & $3(2.4)$ & -- & -- & $2(2.0)$ & $2(18.2)$ & -- & $2(2.8)$ & $2(2.8)$ & -- & $2(2.2)$ & $2(3.8)$ & -- \\
\hline Molluscum contagiosum & 3 & $1(5.3)$ & $2(1.6)$ & -- & -- & $3(3.1)$ & -- & -- & -- & $3(4.2)$ & -- & -- & $3(5.7)$ & -- \\
\hline Candida & 2 & -- & $1(0.8)$ & -- & $1(2.9)$ & $1(1.0)$ & -- & -- & -- & $2(2.8)$ & -- & $2(2.2)$ & -- & -- \\
\hline Candida + Pseudomonas & 1 & -- & $1(0.8)$ & -- & $1(2.9)$ & -- & -- & -- & $1(1.4)$ & -- & -- & $1(1.1)$ & -- & -- \\
\hline Candida + GBS & 1 & -- & $1(0.8)$ & -- & -- & $1(1.0)$ & -- & -- & $1(1.4)$ & -- & -- & $1(1.1)$ & -- & -- \\
\hline Zygomycetes & 1 & -- & $1(0.8)$ & -- & $1(2.9)$ & -- & -- & -- & $1(1.4)$ & -- & -- & $1(1.1)$ & -- & -- \\
\hline Fusarium & 1 & -- & $1(0.8)$ & -- & -- & $1(1.0)$ & -- & -- & $1(1.4)$ & -- & -- & $1(1.1)$ & -- & -- \\
\hline Lice & 1 & -- & $1(0.8)$ & -- & -- & $1(1.0)$ & -- & -- & -- & $1(1.4)$ & -- & $1(1.1)$ & -- & -- \\
\hline Total & 143 & $\begin{array}{c}19 \\
(100.0)\end{array}$ & $\begin{array}{c}124 \\
(100.0)\end{array}$ & & $\begin{array}{c}34 \\
(100.0)\end{array}$ & $98(100.0)$ & $\begin{array}{c}11 \\
(100.0)\end{array}$ & & $72(100.0)$ & $71(100.0)$ & & $0(100.0)$ & $53(100.0)$ & \\
\hline
\end{tabular}

*Fisher's exact test was used for categorical variables.

$P$-values were not calculated for comparisons where one value was equal to 0 or 1 or when both values together were less than or equal to 5 .

Abbreviations: HSV, Herpes simplex virus; VZV, Varicella zoster virus; HPV, Human papilloma virus; GBS, Group B streptococcus; GVHD, Graft-versus-host disease 
Table 3. Infectious class by engraftment period.

\begin{tabular}{|c|c|c|c|c|c|c|c|}
\hline Organism & $\begin{array}{c}\text { Preengraftment }^{3} \\
\text { [1-30 days] } \\
\text { n (column \%) }\end{array}$ & p-value* & $\begin{array}{c}\text { Early postengraftment }^{3} \\
\text { [30-100 days] } \\
\text { n (column \%) }\end{array}$ & p-value ${ }^{\star}$ & $\begin{array}{c}\text { Late postengraftment }^{3} \\
{[>100 \text { days }]} \\
n \text { (column } \%)\end{array}$ & p-value* & $\begin{array}{c}\text { Total, } \mathbf{n} \\
\text { (column \%) }\end{array}$ \\
\hline Herpes (HSV/VZV) & $6(26.1)$ & 0.5769 & $4(44.4)$ & 0.0923 & $20(18.0)$ & 0.1382 & $30(21.0)$ \\
\hline $\begin{array}{l}\text { Unknown Organism- Non Cellulitis } \\
\text { Associated }^{2}\end{array}$ & $6(26.1)$ & 0.2398 & $2(22.2)$ & 0.6569 & $17(15.3)$ & 0.2890 & $25(17.5)$ \\
\hline $\begin{array}{l}\text { Unknown Organism- Cellulitis } \\
\text { Associated }^{2}\end{array}$ & $2(8.7)$ & 0.7387 & $2(22.2)$ & 0.3413 & $15(13.5)$ & 1.000 & 19 (13.3) \\
\hline $\begin{array}{l}\text { Identified Bacterial Pathogen- } \\
\text { Cellulitis Associated }^{1}\end{array}$ & $3(13.0)$ & 0.4399 & $1(11.1)$ & 0.5869 & $9(8.1)$ & 0.4877 & $13(9.1)$ \\
\hline Mixed Bacterial Pathogen ${ }^{1}$ & - & & - & & $10(9.0)$ & & $10(7.0)$ \\
\hline Dermatophyte & $1(4.3)$ & & - & & $4(3.6)$ & & $5(3.5)$ \\
\hline HPV & - & & - & & $4(3.6)$ & & $4(2.8)$ \\
\hline Molluscum & - & & - & & $3(2.7)$ & & $3(2.1)$ \\
\hline Candida & $1(4.3)$ & & - & & $1(0.9)$ & & $2(1.4)$ \\
\hline Candida + Pseudomonas & - & & - & & $1(0.9)$ & & $1(0.7)$ \\
\hline Zygomycetes & - & & - & & $1(0.9)$ & & $1(0.7)$ \\
\hline Fusarium & - & & - & & $1(0.9)$ & & $1(0.7)$ \\
\hline Lice & - & & - & & $1(0.9)$ & & $1(0.7)$ \\
\hline Grand Total & $23(100)$ & & $9(100)$ & & $111(100)$ & & $143(100)$ \\
\hline
\end{tabular}

${ }^{*}$ Fisher's exact test was used for categorical variables.

$P$-values were not calculated for comparisons where one value was equal to 0 or 1 or when both values together were less than or equal to 5 .

Abbreviations: HSV, Herpes simplex virus; VZV, Varicella zoster virus; HPV, Human papilloma virus; GBS, Group B streptococcus 
Additionally, $67.6 \%$ of viral infections occurred when the patient was not neutropenic (25/37). The majority of viral infections involved herpes (HSV/VZV) (30/37). Sixty-six percent of the herpes (HSV/VZV) infections occurred in the late postengraftment period (20/30) (Table 3). Our herpes category included the varicella-zoster virus. A previous study found that varicellazoster virus infections most commonly occur during the late postengraftment period and a few herpes simplex virus infections are seen in the preengraftment period [23]. These previous findings support the data found in this study and also explain why most of the herpes (HSV/VZV) infections occurred with no neutropenia present $(20 / 30,66.7 \%)$ (Table 2).

In this study, more allogeneic transplant patients had at least one clinically diagnosed skin infection compared to autologous transplant patients. However, patients with autologous transplants on average had a higher number of skin infections following transplantation. Additionally, the infectious agents within allogeneic and autologous infections were not necessarily equal in number or class. Herpes (HSV/VZV) caused $28 \%$ of infections associated with allogeneic transplants (25/90) but only $9 \%$ in those with autologous transplants $(5 / 53)$ with a significant $p$-value of 0.0104 . A previous study states that allogeneic transplantation acts as a risk factor for herpes (HSV/VZV) infections, which supports our current findings. However, chronic GVHD does not seem to act as an infectious risk factor for herpes (HSV/VZV) ( $p$ value $=0.0636$ ) [23]. Fungal infections caused by Fusarium, Zygomycetes, and Candida regardless of fungal prophylaxis were only found in patients with allogeneic infections, which is well-reported in current literature [19, 24, 25]. Additionally, only $13 \%$ of infectious agents went unidentified in allogeneic transplant patients $(12 / 90)$, whereas $25 \%$ went unidentified in autologous transplant patients (13/53) (pvalue $=0.1114$ ). Perhaps the clinical manifestations of infections are more severe in those with allogeneic transplants, prompting diagnostic testing.

Patients with GVHD had a lower percentage of unknown infections at $26 \% \quad(19 / 72)$ while patients with no GVHD had 35\% unknown infection (25/71) (Table 2). This could also be due to a more severe clinical manifestation of infections in those with GVHD, prompting more diagnostic testing and, therefore, a higher rate of infectious agent identification.

A higher proportion of infections remained unknown when no dermatology consultation was involved as compared to the cases that did involve a dermatology consultation. The clinical manifestations shown in cases that did receive a dermatology consult may have been more worrisome compared to those infections that did not receive a dermatology consultation. A study found that nearly half $(47 \%)$ of cancer patients requiring dermatologic consultation had a hematologic malignancy [26]. Additionally, dermatology consultations have been associated with better outcome in hospitalized patients, including shorter lengths of stay [27].

More tests were ordered when the infection had a dermatology consultation as opposed to when there was none and diagnostic tests were not necessarily ordered by the dermatologist. Therefore, dermatologists are not necessarily ordering more diagnostic tests compared to other doctors involved with HSCT patients. We believe that some infections remained unidentified or unresolved despite multiple diagnostic tests, which then prompted a dermatology consultation, resulting in a higher average number of diagnostic tests. A study found that infections that received a dermatology consultation generally did not require further lab testing [26], making our belief plausible.

Though limited by small sample size and no control group, we conclude that the etiologies and severities of clinically identified skin infections in HSCT patients are varied and require continuous vigilance of dermatological health. Additionally, the study is also limited in that the "infections" were clinically identified. It must be understood that health professionals treated these skin pathologies as "infections" whether or not an infectious etiology was explicitly identified. Therefore, there is the possibility that these pathologies were misdiagnosed and/or mistreated as infections. Nevertheless, the goal of this study was to better characterize the clinical management of these clinically identified pathologies. Dermatology 
consultations are utilized less often than expected. Therefore, being aware of a patient's infectious characteristics and understanding clinical manifestations and management may help healthcare providers better treat dermatological infectious complications.

\section{ACKNOWLEDGMENTS}

This study has been presented at American Academy of Dermatology Annual Meeting, San Diego, California, February 16-20, 2018 and Society of Hospital Medicine Annual Meeting, Orlando, Florida, April 9-11, 2018.

Declaration of Conflicting Interests: The authors declare that they have no conflict of interest.

Funding source: Not declared.

\section{REFERENCES}

1. Gil L, Styczynski J, Komarnicki M. Infectious Complication in 314 Patients after High-Dose Therapy and Autologous Hematopoietic Stem Cell Transplantation: Risk Factors Analysis and Outcome. Infection 2007; 35:421-7.

2. Solberg CO, Meuwissen HJ, Needham RN, Good RA, Matsen JM. Infectious complications in bone marrow transplant patients. Br Med J 1971; 1:18-23.

3. Winston DJ, Gale RP, Meyer DV, Young LS. Infectious complications of human bone marrow transplantation. Medicine (Baltimore) 1979; 58(1):131.

4. Holowiecki J. Indications for hematopoietic stem cell transplantation. Pol Arch Med Wewn 2008; 118:658-663.

5. Gardner SL. Application of stem cell transplant for brain tumors. Pediatr Transplant. 2004; 8:28-32.

6. Parody R, Martino R, Rovira M, et al. Severe Infections after Unrelated Donor Allogeneic Hematopoietic Stem Cell Transplantation in Adults: Comparison of Cord Blood Transplantation with Peripheral Blood and Bone Marrow Transplantation. Biol Blood Marrow Transplant 2006; 12:734-748.

7. Bangert C, Brunner PM, Stingl G. Immune functions of the skin. Clin Dermatol 2011; 29:360376.

8. Salmon JK, Armstrong CA, Ansel JC. The skin as an immune organ. West J Med. $1994 ; 160: 146$.

9. Bangert C, Brunner PM, Stingl G. Immune functions of the skin. Clin Dermatol 2011; 29:360376.

10. Salmon JK, Armstrong CA, Ansel JC. The skin as an immune organ. West J Med 1994;160:146.
11. Klingspor L, Saaedi B, Ljungman $P$, Szakos A. Epidemiology and outcomes of patients with invasive mould infections: a retrospective observational study from a single centre (20052009). Mycoses 2015; 58:470-477.

12. Bishop MR, editor. Hematopoietic Stem Cell Transplantation. Boston, MA: Springer US; 2009. http://link.springer.com/10.1007/978-0-387-78580-6. Accessed 22 Dec 2016.

13. Amir G, Salant $H$, Resnick IB, Karplus R. Cutaneous toxoplasmosis after bone marrow transplantation with molecular confirmation. J Am Acad Dermatol 2008; 59:781-784.

14. Komitopoulou A, Tzenou T, Baltadakis J, Apostolidis J, Karakasis D, Harhalakis N. Is leishmaniasis an "unusual suspect" of infection in allogeneic transplantation? Transpl Infect Dis 2014; 16:1012-1018.

15. Biesbroeck LK, Shinohara MM. Inpatient Consultative Dermatology. Med Clin North Am 2015; 99:1349-1364.

16. Song $H$, Robinson SN, Huang JT. Outpatient dermatology consultation impacts the diagnosis and management of pediatric oncology patients: A retrospective study. J Am Acad Dermatol 2017; 77:879-885.

17. Afsar F. Analysis of pediatric dermatology inpatient consultations in a pediatric teaching hospital. Arch Argent Pediatr 2017; 115.

18. Harris PA, Taylor R, Thielke R, Payne J, Gonzalez $\mathrm{N}$, Conde JG. Research electronic data capture (REDCap)—A metadata-driven methodology and workflow process for providing translational research informatics support. J Biomed Inform 2009; 42:377-381.

19. Auner $\mathrm{H}$, Sill $\mathrm{H}$, Mulabecirovic A, Linkesch $\mathrm{W}$, Krause $R$. Infectious complications after autologous hematopoietic stem cell transplantation: comparison of patients with acute myeloid leukemia, malignant lymphoma, and multiple myeloma. Ann Hematol 2002; 81:374-377.

20. Mays SR, Bogle MA, Bodey GP. Cutaneous fungal infections in the oncology patient. Am J Clin Dermatol 2006; 7:31-43.

21. Fourie R, Ells R, Swart CW, Sebolai OM, Albertyn $\mathrm{J}$, Pohl $\mathrm{CH}$. Candida albicans and Pseudomonas aeruginosa Interaction, with Focus on the Role of Eicosanoids. Front Physiol. 2016; 7:64.

22. McCarty JM, Haber J. Group B streptococcal soft tissue infections beyond the neonatal period. West $\mathrm{J}$ Med. 1987; 147:558.

23. Sahin U, Toprak SK, Atilla PA, Atilla E, Demirer T. An overview of infectious complications after allogeneic hematopoietic stem cell transplantation. J Infect Chemother 2016; 22:505-514. 
24. Salazar R, Sola C, Maroto P, et al. Infectious complications in 126 patients treated with high-dose chemotherapy and autologous peripheral blood stem cell transplantation. Bone Marrow Transplant 1999; 23:27-33.

25. Kolbe K, Domkin D, Derigs HG, Bhakdi S, Huber C, Aulitzky WE. Infectious complications during neutropenia subsequent to peripheral blood stem cell transplantation. Bone Marrow Transplant 1997; 19:143-147.

26. Phillips GS, Freites-Martinez A, Hsu M, et al. Inflammatory dermatoses, infections, and drug eruptions are the most common skin conditions in hospitalized cancer patients. J Am Acad Dermatol 2018; 78:1102-9.

27. Milani-Nejad $\mathrm{N}$, Zhang $\mathrm{M}$, Kaffenberger $\mathrm{BH}$. Association of Dermatology Consultations with Patient Care Outcomes in Hospitalized Patients with Inflammatory Skin Diseases. JAMA Dermatol 2017; 153:523. 